\title{
Integrated management system of Electronic safety based on Gray correlation
}

\author{
Jianjun Shi ${ }^{1, a}$, Chao Zhang ${ }^{1, b}$, Xiaqing Liu $^{2,3, c}$ \\ ${ }^{1}$ School of Business, University of International Business and Economics, Beijing 100029, China \\ ${ }^{2}$ Department of College English, Shandong Institute of Business and Technology, Shandong 264005, \\ China \\ ${ }^{3}$ College of Economics and Management, Nanjing University of Aeronautics and Astronautics, \\ Jiangsu 211106, China

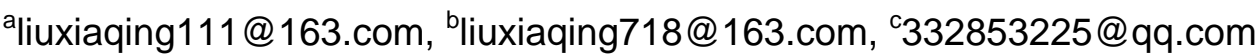

Keywords: Integrated electronic system; Security management; ASAAC model.

\begin{abstract}
The coming of information age makes the integrated electronic system in industrial field face new challenges. This paper takes the security management system of electronic railway industry the industrial area as the researching object, discussing the architecture of ASAAC standard system, by means of gray correlation analysis method, it puts forward ideas of assessment on the system safety.
\end{abstract}

\section{Introduction}

The architecture of software can be defined as IMA (Integrated Modular Avionics) in railway electronic system, which can provide a uniform requirement for the design and development of the software framework of the core processing system. The software framework of ASAAC standard system is based on the architecture of the layered software, the structure of this layered system consists of three layers, including application layer (AL), operating system layer (OSL) and module of supporting layer (MSL). Application layer defines the function application as well as the management application. The function application is usually so-called application task; application management is the implementation of the system management which is closely related to the application. The operating system layer defines three parts, namely, the operating system, general management system (GSM) and running blueprint. General management system includes health monitoring, fault management, configuration management and security management; operating blueprint contains all the information of the configuration and the core part of management system. Module supporting layer defines the basic drive management of the low-level hardware resources. The interface between the application layer and operating system layer is APOS, while the interface between the operating system layer and supporting layer is MOS. These interfaces make the three levels be independently separated with each other.

\section{The Architecture Component of System Software}

The software architecture of ASAAC standard system defines a set of components and interfaces, moreover, the interface of software can be divided into two categories, namely, direct interface and logical interface. In the direct interface, it defines the application layer and the interface of operating system layer (APOS), module support layer / module operating system layer (MOS), system management (SMBP) / interface of blueprint as well as system management / interface of operating system (SMOS), totally four kinds of interfaces. While in the logic interface, it defines four kinds of interface, namely, the logic interface of the operating system (OLI), the logic interface of general system management (GLI), the logic interface of system management (SMLI) as well as the logic interface of module (MLI). The architecture model of ASAAC software in detail can be shown in Fig. 1: 


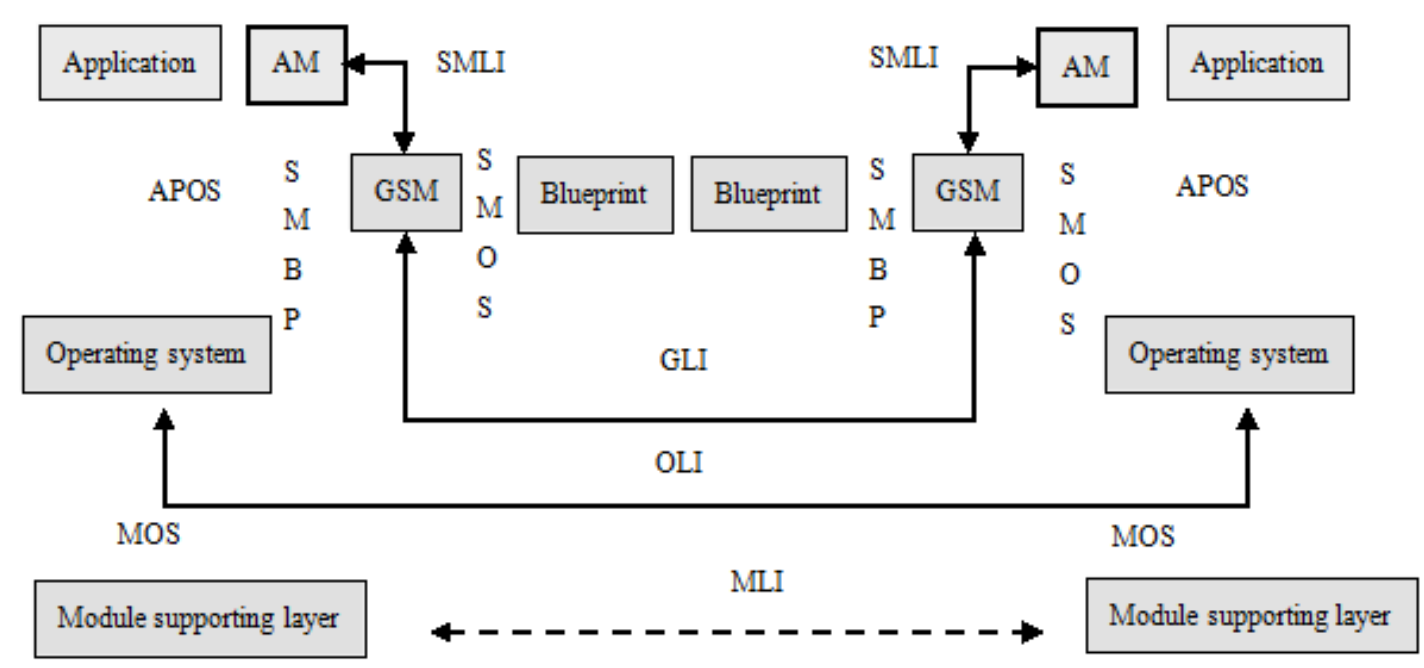

Fig.1 The Architecture module of software system

Application management (AM): it can be responsible for non-standard system management, implementing mission, management mode, the interface between AM and GSM is the logic interface for the system management;

Operating system (OS): it is a specific section that can offer OSL function, which also can control the real-time behavior of the processing unit as well as its related components;

General system management (GSM): it is responsible for the core processing management, which can be including four parts: health monitoring (HM), (FM) fault management, configuration management (CM) and safety management (SM);

Running blueprint (RTBP): it contains the information of the configuration and management at the core process on the host computer (such as: processing description, routing information, fault management, etc.);

Module of supporting layer (MSL): it encapsulates the underlying hardware details, which can provide methods of the universal, independent access to the underlying resources;

The interface from application to the operating system (APOS): the separated aircraft depends on software named as AL, as well as the independent software of aircraft named as OSL. Its purpose is to provide a standard OS service interface for the AL process, which can improve the re-usability and portability of the application software;

The interface from module support to operating system (MOS): in order to divide the OSL and hardware, it needs software called MSL. Its purpose is to provide a transparent interface for operating system which can independently adjust the function of hardware. Thus, MOS allows the same OSL software reside in the specific CFM, regardless of the underlying hardware;

The interface from safety management to the blueprint (SMBP): the interface between GSM and the blueprint is encapsulated in OSL, which can allow and the implementation and structure of blueprint is non - standard, but it should be the interface from the defined blueprint to the standard interface of GSM;

The interface from system management to the operating system (SMOS): it is packaged in OSL, which can describe the services for GSM offered by the operating system;

The logic interface of operating system (OLI): it describes the process with two examples of OS, as well as the usage of VC communication;

The logic interface of GSM (GLI): it describes mutual communication between two examples of GSM, moreover, the internal communication between GSM is hierarchical;

The logic interface of system management (SMLI): it describes communication protocol between AM and GSM based on VC. AM and GSM must be cooperated with each other, with the help of SMLI for communication and synchronization;

The logic interface of module (MLI): it defines the interface between the modules and the system structure of the interface to meet the demand of interoperability. 


\section{System Management}

System management is a whole management system from the flight of the aircraft to landing stage, which is composed of two parts, namely, the application management of application layer that is located in ASAAC model of the three layer stack (TLS), as well as the general system management GSM of the operating system layer. Its main tasks can be included: the initialization of controlling system, reconfiguration, power off; authentication, filtering, fault locating; providing related security services.

\section{The Main Functions of Each Management Level of the System}

AC. AC is a single entity of the system management, which is responsible for controlling and monitoring the entire integrated modular railway electronic system (IMA), as well as responsible for loading the system data (both task data and safety data); AC is responsible for the initialization of system security. Before the operation of the system, AC is responsible for the initialization of IA and $\mathrm{RE}, \mathrm{AC}$ can receive the initialization security request and key request of IA and RE.

IA. IA is a logical group of comprehensive application. This group is a dynamic group, which can be generated dynamically according to the need of the task; each IA can control one or more RE; the internal of IA may be organized as a hierarchical layer, such as: a comprehensive district may be included one or more levels of IA, in this case, IA in the lower layer must control one or more PE; the railway electric system can be designed a system without including IA level, in this case, it can exist one $\mathrm{AC}$ and one or more RE.

RE. RE is the lowest layer, which is responsible for dealing with a single PE; it can receive the security information of initialization from CM, at the same time, it can send the initialization security request and key request to the upper layer.

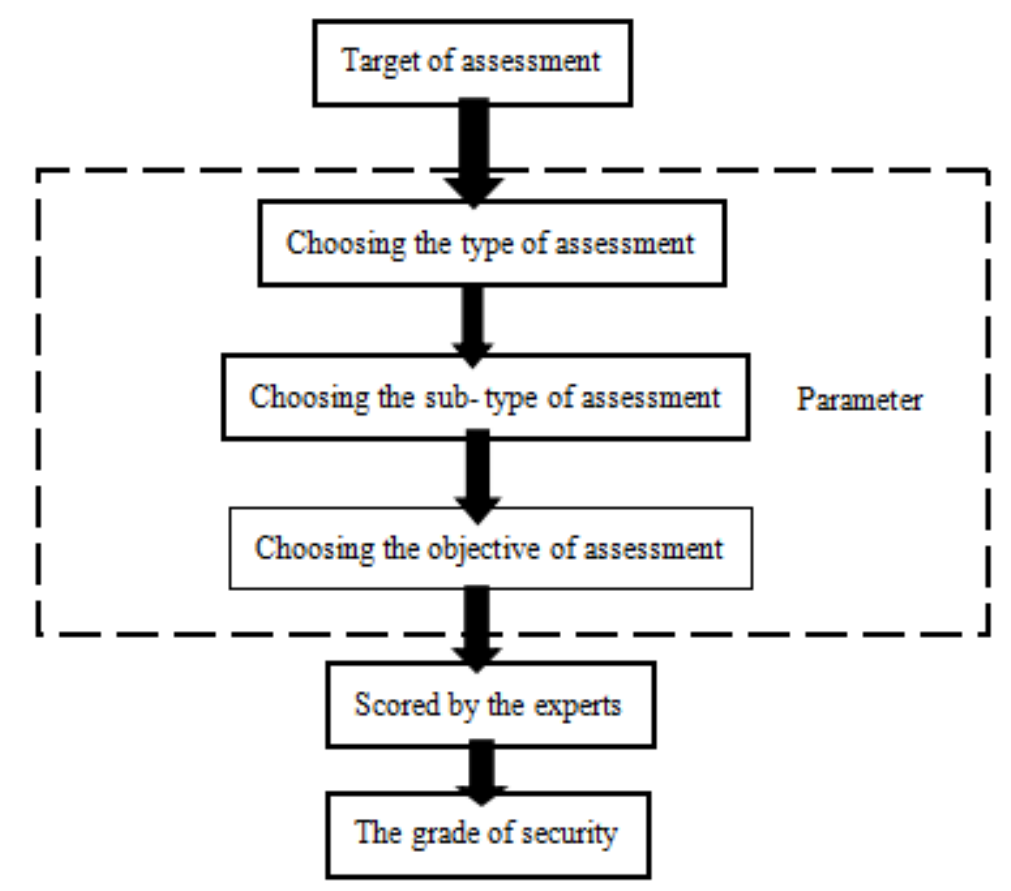

Fig. 2 The model diagram of security assessment

\section{Gray Correlation}

The introduction of GRAP method is in order to finally get the security grade of the targeted information system. $\omega$ Can represent the fitness of the security grade, while $\omega_{i k}$ can represent that the possibility of component $k$ with the fitness of the security grade is $i$. supposing there are $n$ components, the fitness of the security grade of these safety $n$ components can consist the following matrix: 


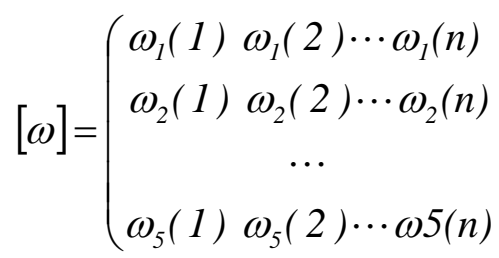

The concrete steps of GRAP are as follows:

(1) Getting series with priority value $\omega_{0}$

$$
\omega_{0}=\left\{\omega_{0}(k)(k=1,2, \cdots, n)\right\}
$$

The formula $\omega_{0}(k)$ can represent the maximum value of all the possibilities of security of component $k$, namely,

$$
\omega_{0}(k)=\max \left(\omega_{1}(k), \omega_{2}(k), \omega_{3}(k), \omega_{4}(k), \omega_{5}(k)\right)
$$

The process of obtaining the most appropriate security level is to obtain the optimal value of fitness evaluation. This paper uses the GRAP method to get the appropriate security grade of the security rating of various components.

\section{Conclusion}

With the development of railway electronic system, the railway electronic system for next generation can present integrated feature, modular feature, complicated feature and so on, which also has the characteristics with high degree of resource sharing, high degree of information fusion and highly intensive degree of software, compared with the independent type of railway electronic system.Each module of the integrated railway electronic system can share the interactive resources together and complete the common task together, therefore, the problem of information security has become one of the main challenges that it should face.

\section{References}

[1]Christopher B. Watkins. 2006, Integrated Modular Avionics: Managing the Allocation of Shared Intersystem Resources. 25th Digital Avionics Systems Conference, 2006 IEEE/AIAA. vol.8, p1-12.

[2]Graham Jolliffe. 2005, Producing a safety case for IMA blueprints. Digital Avionics Systems Conference, DASC 2005. vol.24, p11-14.

[3]Ames, B. 2003, ”Real-Time Software Goes Modular”, Military \& Aerospace Electronics, vol.14, pp24-29.

[4]Rushby, J. and B. Randell. 1983, “A distributed secure system,” IEEE Computer, vol.16, p55-67. 